\title{
Enhancement of Elastic Modulus of Epoxy Resin with Carbon Nanotubes
}

\author{
Vijay Kumar Srivastava \\ Department of Mechanical Engineering, Indian Institute of Technology, Varanasi, India \\ E-mail: vijayks210@gmail.com \\ Received February 1, 2011; revised March 4, 2011; accepted March 10, 2011
}

\begin{abstract}
:
Nanocomposites consisting of multiwall carbon nanotubes (MWCNT) and epoxy resin were produced by a standard calendaring technique. In this study, 3\% multiwall carbon nanotube particles were dispersed in epoxy resin by weight to produce the multiwall carbon nanotubes/epoxy composite. Nanohardness and Raman spectroscopy tests were used to obtain the modulus of elasticity and Raman intensity of MWCNTs/ epoxy resin composite. The results show that the Raman intensity increased with the increase of Raman shift and Raman intensity also affected with the reinforcement of multiwall carbon nanotubes and $1 \%$ exposure of laser power. Also, nanohardness increased with increase of modulus of elasticity, which indicated that the toughness of epoxy resin improved with the addition of multiwall carbon nanotubes.
\end{abstract}

Keywords: Epoxy Resin; Multiwall Carbon Nanotubes; Nanohardness; Raman Spectroscopy; Modulus of Elasticity

\section{Introduction}

Iijima discovered carbon nanotubes (CNT) in 1991 [1]. Single-walled carbon nanotubes (SWCNT) are fullerenebased structures that can be viewed as a single sheet of graphite rolled into a cylinder several microns in length and $1 \mathrm{~nm}$ in diameter [2]. Multiwalled carbon nanotube (MWCNT) are similar to SWCNT but with many layers of graphite sheets in the cylinder structure. MWCNT are several microns in length and $5-50 \mathrm{~nm}$ in diameter depending on the number of layers. Due to their graphitelike structure, CNT are expected to have excellent mechanical properties. Lu [3] adopted an empirical lattice dynamics model to predict the elastic properties of CNT. His study suggested that the elastic properties of SWCNT are not sensitive to size and chirality and predicted the Young's modulus to be $1 \mathrm{TPa}$, the shear modulus to be $\sim 0.45 \mathrm{TPa}$, and the bulk modulus to be $\sim 0.74 \mathrm{TPa}$. $\mathrm{Yu}$ et al [4] used an atomic force microscope (AFM) to measure the mechanical properties of MWCNT and found the tensile strength and modulus to be in the range of 11-63 GPa and 270-950 GPa respectively.

Their excellent mechanical properties and superior length to diameter ratio make CNT very attractive for composite reinforcement. Recent research on nanotubebased composites has focused on polymer matrix compo- sites. Qian et al [5] conducted an experimental study and showed that the addition of $1 \mathrm{wt} \%$ CNT resulted in a 36$42 \%$ increase in the elastic stiffness and a $25 \%$ increase in the tensile strength for polystyrene (PS) -based composites. They observed CNT pull-out in the transmission electron microscope (TEM) that suggested CNT were able to bridge the crack in the PS matrix and serve as reinforcements in composites. In order to fully utilize the extraordinary mechanical properties of CNT, the strong interfacial bonding between CNT and the polymer matrix is very critical. Wanger [6] obtained the fibre-polymer interfacial shear strength to the critical aspect ratio, to predict the CNT-polymer interfacial shear strength by using the critical length from 100 to $500 \mathrm{~nm}$. Wagner calculated the CNT-polymer interfacial bond strength to be in the range of 50-250 MPa. Lio and Li [7] performed molecular mechanics simulations and elasticity calculations to predict the interfacial characteristics of a CNTPS composite system. They simulated the pull-out of CNT from the matrix and calculated the CNT-PS interfacial fibre-polymer shear strength of high modulus carbon fibres in epoxy matrix usually ranges from $50-100 \mathrm{MPa}$, it was concluded that a CNT-polymer can achieve more efficient interfacial stress transfer than current advanced composites. In addition to the improvement in tensile strength, it was reported that Vickers hardness of epoxy 


\section{K. SRIVASTAVA}

increased by $20 \%$ with an additional $2 \mathrm{wt} \%$ CNT [8-12].

It is evident that CNT can be potentially used to reinforce the polymer and improve the mechanical properties. However, no experiment has come ever demonstrated a CNT based composite with better performance than current advanced polymer composites. For further advances in this area, researchers pointed out that several critical issues such as improvement in polymer interfacial bonding, MWCNT interwall sliding under tension, CNT dispersion and alignment and polymer matrix shrinkage during the process must be addressed [11]. These issues may contribute to the uncertainty of manufacturing CNT/polymer composites with desired characteristics.

Raman spectroscopy has historically played an important role in the study and characterization of graphite materials, being widely used over the last four decades to characterize pyrolytic graphite, carbon fibres, glassy and carbon nanotubes [12]. For $\mathrm{sp}^{2}$ nanocarbons such as Graphene and carbon nanotubes, Raman spectroscopy can give complete information about crystallite materials. In this article, Raman spectroscopy, nanohardness and scanning electron microscopy were used to see the effect of multiwall carbon nanotubes in the elastic modulus of epoxy resin. Our hypothesis is that the CNT will serve as an excellent reinforcement to toughen the epoxy resin due to its small scale, excellent mechanical properties and good chemical compatibility with the composite adherends.

\section{Experimental Details}

Araldite, LY-556 (55\%), hardener, HY-917 (49\%) and accelerator, DY-070 $(0.28 \%)$ were used as epoxy resin. $3 \%$ multiwall carbon nanotubes filled epoxy resin (LY556) were produced using a lab-scale three-roll-mill (Exakt $120 \mathrm{E}$ ), which enables the introduction of very high shear forces (up to 200,000 s $\mathrm{s}^{-1}$ ) throughout the suspension. The pre-dispersed suspension was then given batchwise onto the roll with dwell times of $2 \mathrm{~min}$. The dispersive forces on the suspension were acting in the gap $(5 \mu \mathrm{m})$ between the rolls. After dispersion of the nanoparticles in the resin LY-556, the hardener and accelerator are usually added in a vacuum dissolver in order to avoid trapped air in the suspension. Then the mixture was placed in a vacuum chamber for $20 \mathrm{~min}$ to eliminate the bubbles introduced during the rolling process.

Raman spectroscopy is nondestructive and readily available and measurements can be made over a wide range of temperature or pressures. It can provide unique information about vibrational and electron properties of the material. Even though it is not a direct method, it can also be used to determine the structure of the material and allows the identification of materials through the characteristics vibrations of certain structures. Because the Raman intensity of a vibration in a crystal depends on the relative directions of the crystal axis and the electric wave polarization of the incident and scattered light. Therefore, Raman spectroscopy was used to determine the differences in Raman intensity of epoxy resin, MWCNTs/epoxy resin composite and $1 \%$ laser power exposed MWCNTs/epoxy resin composite.

The indentation method has become a standard way to measure the mechanical properties of thin-film and smallscale structures. A depth-sensing indenter, i.e. nanoindenter, can measure the indentation displacement $(h)$ and elastic contact stiffness $(S)$ during a programmed indentation loading process, where $S$ is defined as:

$$
S=\frac{\mathrm{d} P}{\mathrm{~d} h_{c}}
$$

where $P$ is the indentation load, and $h_{c}$ is the elastic component of $h$. Using the data analysis method proposed by Oliver and Pharr [13], the contact depth, $h_{c}$, can be estimated by

$$
h_{c}=h-€ \frac{P}{S}
$$

where $€$ is a constant, 0.75 . Then, based on the predetermined indenter tip geometry, we can calculate the projected contact area $(A)$ from $h_{c}$. Finally, the elastic modulus $(E)$ and the hardness $(H)$ of materials can be calculated by

$$
\begin{gathered}
E_{r}=\frac{\sqrt{J I}}{2} \frac{S}{\sqrt{A}} \\
H=\frac{P}{A}
\end{gathered}
$$

Here $E_{r}$ is the reduced modulus defined as

$$
E_{r}=\frac{1-\mu^{2}}{E}+\frac{1-\mu_{i}^{2}}{E_{i}}
$$

where $E$ and $\mu$ are Young's modulus and Poisson's ratio of the indented material respectively; and $E_{i}$ and $\mu_{i}$ are the corresponding values of the indenter tip. $\mathrm{H}$ is the mean pressure under the indenter.

The nanohardness and the elastic modulus of the epoxy resin and MSCNTs/epoxy resin composite were determined using a Nano Indentation tester (CSM instrument). A triangular pyramid Berkovich indenter was used, its indent shape and side view angles were 65.3 and 77.05 respectively. The poisson's ratio of the samples were estimated as $\mu=0.3$, because in the calculations of elastic modulus, an error in the estimation of the Poisson ratio does not produce a significant effect on the resulting value of the elastic modulus. Three indentations were carried out to depth of $1000 \mathrm{~nm}$ where the indentation was kept for $10 \mathrm{~s}$ before unloading. The loading and the unloading rate were $10 \mathrm{mN} \mathrm{min}$.

Finally, scanning electron microscopy was used to identify the effect of MWCNTs in the epoxy resin. 


\section{K. SRIVASTAVA}

\section{Results and Discussion}

In this study MWCNT were dispersed by three mill rolling machine in an epoxy resin, with the aim of improving mechanical properties of MWCNTs/epoxy resin composite. First of all the effect of the presence of MWCNT in the epoxy resin was investigated by Raman spectroscopy technique. Figure 1 shows the Raman spectrum of epoxy resin, MWCNT/epoxy resin and 1\% laser power exposed MWCNT/epoxy resin. These spectral features are clearly distinguishing the variation in Raman intensity. Because, when the bond lengths and angles of graphene are modified by strain, caused by the interaction with a substrate or with other graphene layers or due to external perturbation, the hexagonal symmetry of graphene is broken. It was observed that the Raman spectra intensity increases with increasing the shift angle up to $300 \mathrm{~cm}^{-1}$ and gradually decreases with increasing of shift angle after $400 \mathrm{~cm}^{-1}$. These spectral features are similar for epoxy resin, MWCNTs/epoxy resin and 1\% exposed laser power MWCNTs/epoxy resin composites. Eight peak signal bands are identified from epoxy resin sample at different Raman shift values; 620, 835, 1140, 1240, 1460, $1600 \mathrm{~cm}^{-1}$. The height of these eight signals is gradually reduced with the addition of MWCNTs and exposure of laser power of epoxy resin, indicating a dilution effect of the MWCNTs when blended with epoxy resin. It is believe that epoxy resin exerts a pressure on the individual tubes, which leads to an increase of the breathing mode frequencies. Therefore bands are highly sensitive to microstructure effects and can be used to probe any modification to the flat geometric structure of resin. The microstructure effects induced by multiwall carbon nanotubes or by exposure of laser power. This shows the effect of CNTs and laser power exposure on epoxy resin, because, the interaction between nanotubes and resin polymer is reflected by a peak shift or peak width change. Visible change in the epoxy resin peak locations as a result of the insertion of nanotubes could be detected.

The Raman intensity can vary when multiwall nanotubes interact with elements; this can be used to examine the structure of the interface and obtain information about the nature, localization and force of the interaction. After the nanotubes were dispersed in epoxy resin, Raman intensity was observed towards lower intensities, evidence that MWCNTs were no longer in direct contact with one another tubes. Also, Raman intensities were decreased with the exposure of laser power. These show that Raman spectroscopy is a useful and reliable tool for the investigation of nanotubes dispersion in epoxy resin.

The influence of scratch load on mechanical response of multiwall carbon nanotubes in epoxy resin was investigated by nanoindentation test. Nanohardness and elastic modulus patterns of the epoxy resin and MWCNT/epoxy resin specimens are reported in Figure 2. The results show that the nanohardness increases with increase of elastic modulus. MWCNTs filled epoxy resin gives higher value than the epoxy resin, because MWCNTs improve the mechanical properties of epoxy resin [5-7]. Based on the experimental observation, one can derived that following expression to obtain modulus of elasticity from nardness,

$$
E=4.9+0.86(H)^{1 / 2}
$$

where $E$ and $H$ are the elastic modulus and nanohardness.

The micrograph shows the dispersion of MWCNT in an epoxy resin area as can be seen in Figure 3. Only few small aggregates are remaining, which are smeared out and well penetrated by the epoxy resin matrix. MWCNT/ epoxy resin composite containing 3\% MWCNT exhibit a significant increase in fracture toughness and strength, as well as an enhancement of stiffness, due to resistance of cracks propagation [8], as can be identified from Figure 4. It is also clear that CNT particles resist the formation of crack path due to increase of toughness of MWCNT filled epoxy resin. The mechanisms of increasing the fracture toughness of polymers due to incorporation of particles have been extensively studied within the last three decades [14]. The application of micro-particles exhibits the highest effect in brittle matrix systems. A clear difference in the distribution pattern and agglomerate sizes can be seen between MWCNT and epoxy resin and their interface appears to be much more homogeneous, suggesting a greater dispersion. Figure 5 shows that MWCNT particles strongly bonded with the epoxy resin and it appears like sprouts, because of toughening effects of particle [7]. The epoxy resin is modified by the addition of MWCNT, which participates in minimizing the crack initiation or the propagation by crack blocking or bridging, as can be identified in Figure 4. The extensive MWCNT bridging seen in micrographs are well in agreement with the prior explained mechanisms, MWCNT-bridging effect enhanced the fracture toughness [6].

The most important micro-mechanical mechanism leading to an increase in fracture toughness are due to localized inelastic matrix deformation and void nucleation, particle/fibre debonding and deformations [5]. The characteristics of the matrix polymer are also important for the reinforcing effect of nano-scaled fillers. In general, the plastic zone size of brittle epoxy resin is relatively small. When a resin is filled with nano-particles, a significant amount of particles occur in the plastic zone, while in a composite with micro-particles, only a minor number of them are involved in the plastic zone deformation process. This is clear evidence that the main fracture mechanical mechanism is related to the enormous surface 


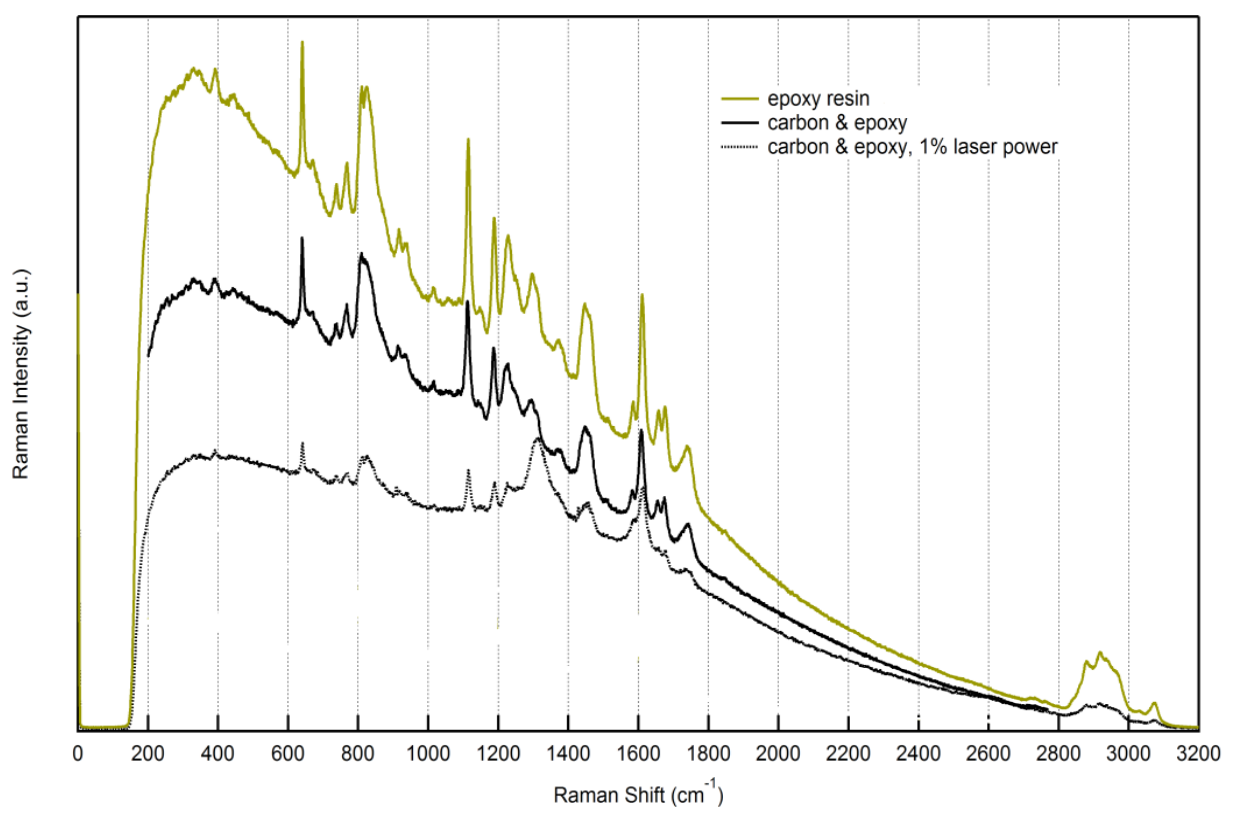

Figure 1. Variation of Raman intensity versus Raman shift from (a). epoxy resin, (b). MWCNTs/epoxy resin and (c). 1\% laser power exposed MWCNTs/epoxy resin.

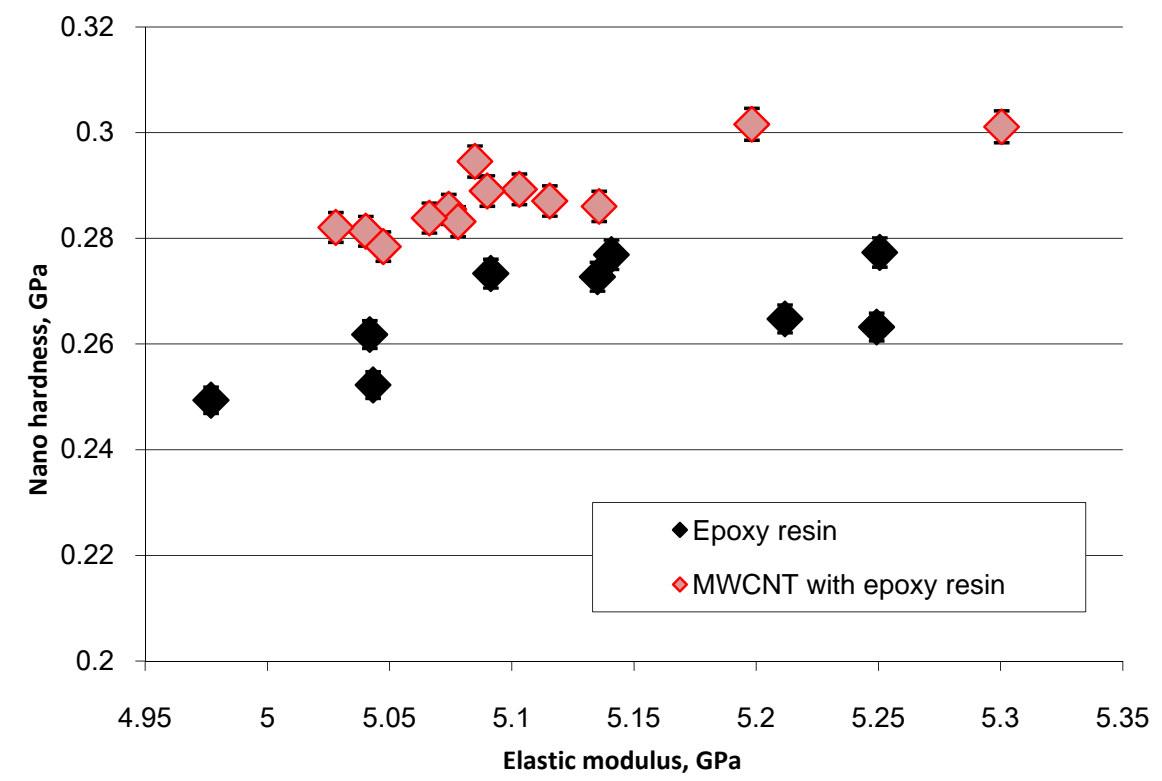

Figure 2. Variation of nanohardness with elastic modulus of epoxy resin and MWCNTs/epoxy resin composites.

area of nano-particles in general. Since, all composites exhibited a partly agglomerated dispersion of the filler, leading to increase in the toughness can be expected to localized inelastic matrix deformation, void nucleation and crack deflection at the agglomerates [7]. Therefore, it is clear from micrographs that resin rich area fractured due to the appearance of shear stress, which increases the adhesive bond strength of ceramic composites. This can be explained by the higher surface area of the double wall carbon nanotubes, which may result in a better load transfer efficiency at the interface region as well as amine functional groups over CNTs which is supposed to promote the dispersion and pronounced covalent bonding to some extent.

\section{Concluding Remarks}

In this article, the effect of multiwall carbon nanotubes in epoxy resin was characterized by the Raman spectroscopy and nanohardness indentation methods. The elastic 


\section{K. SRIVASTAVA}

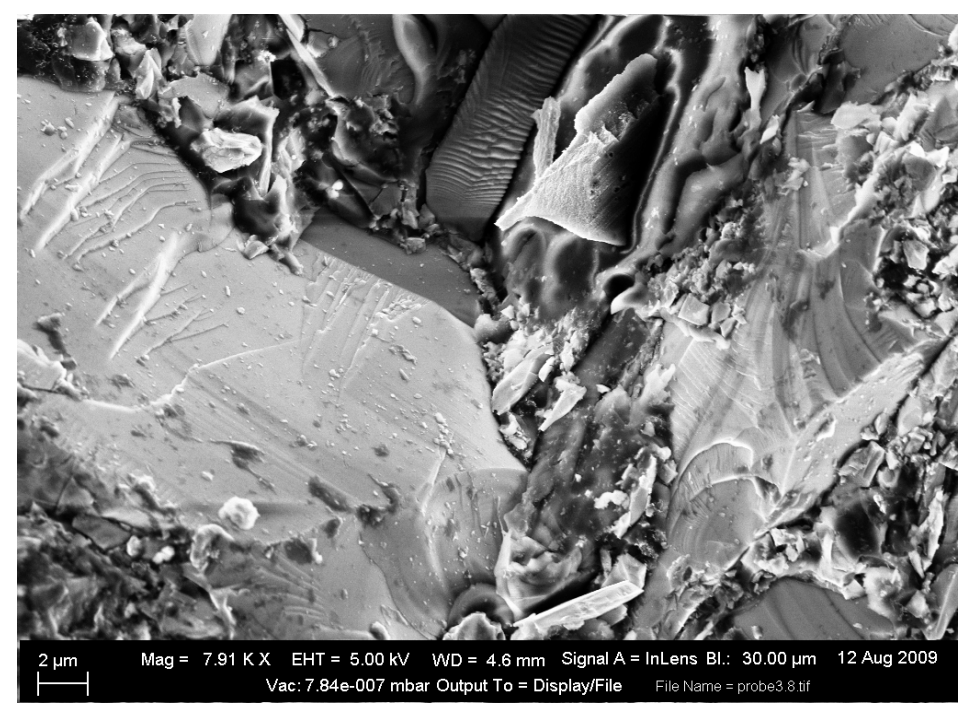

Figure 3. SEM micrograph showing the dispersion of MWCNT particles in the resin rich area.

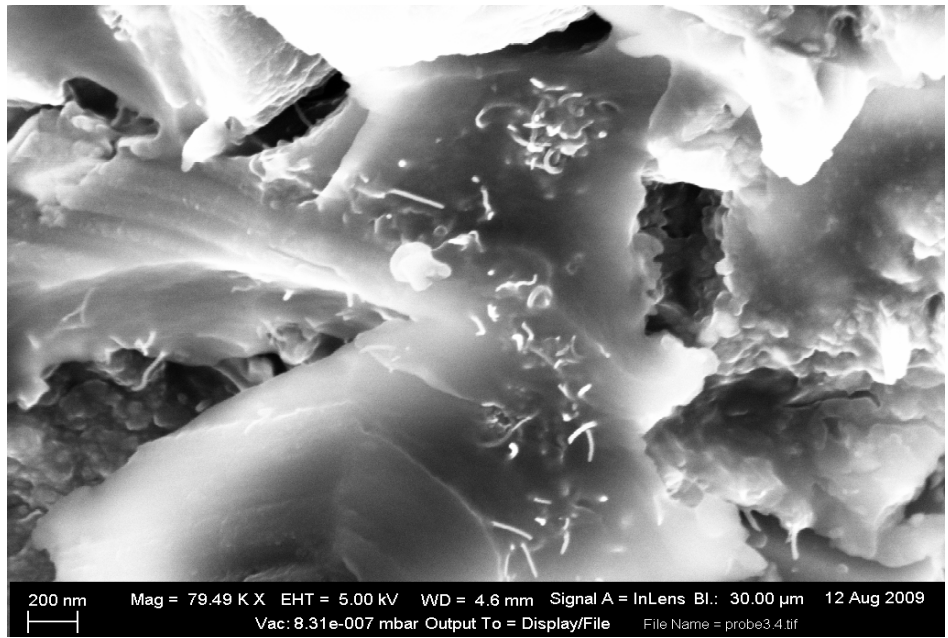

Figure 4. SEM micrograph showing the enhancement of cracks path with MWCNT particles.

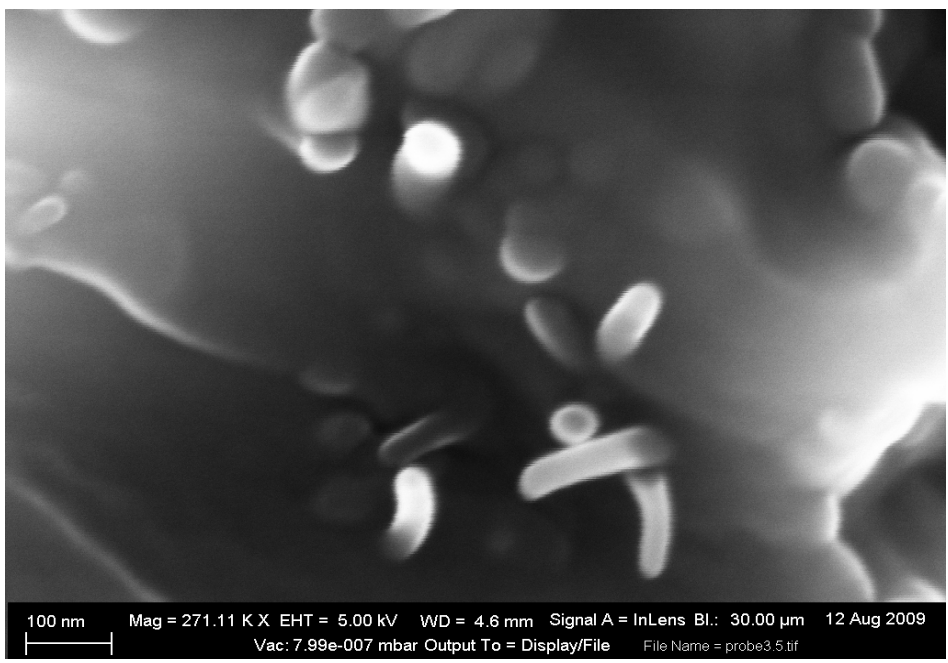

Figure 5. SEM micrograph shows the interface bond of MWCNTs with resin. 
modulus and nanohardness can be related as $E=4.9+0.86(H)^{1 / 2}$. Also, it was found that $3 \mathrm{wt} \%$ MWCNT loading showed good dispersion capability in the epoxy resin, which increases the elastic modulus of neat epoxy resin up to $15 \%$. However, elastic modulus increases with increase of nanohardness, whereas Raman intensity reduces abruptly with the inclusion of MWCNTs and exposure of $1 \%$ laser power.

\section{References}

[1] S. Iijima, "Helical Microtubules of Graphite Carbon," Nature, Vol. 354, No. 7, 1991, pp. 56-58. doi:10.1038/354056a0

[2] P. M. Ajayan, L. S. Schadler, C. Giannaris and A. Rubio, "Single Walled Carbon Nanotube Polymer Composites: Strength and Weakness," Advanced Materials, Vol. 12, No. 10, 2000, pp. 750-753. doi:10.1002/(SICI)1521-4095(200005)12:10<750::AIDADMA750>3.0.CO;2-6

[3] J. P. Lu, "Understanding of Carbon Nanotubes: From Basic to Applications," Journal of Physics and Chemistry of Solids, Vol. 58, No. 11, 1997, pp. 1649-1655. doi:10.1016/S0022-3697(97)00045-0

[4] M. F. Yu, O. Lourie, M. Dyer, K. Moloni, T. F. Kelly and R. S. Ruoff, "Strength and Breaking Mechanism of Multiwalled Carbon Nanotubes under Tensile Load," Science, Vol. 287, No. 10, 2000, pp. 637-643. doi:10.1126/science.287.5453.637

[5] D. Qian, E. C. Dickey, R. Andrews and T. Rantell, "Load Transfer and Deformation Mechanisms in Carbon Nanotubes-Poly-Styrene Composites," Applied Physics Letters, Vol. 76, No. 20, 2000, pp. 2868-2670. doi: $10.1063 / 1.126500$

[6] H. D. Wanger, "Nano Composites: Issues at the Interface," Materials Today, Vol. 7, No. 11, 2004, pp. 38-42. doi:10.1016/S1369-7021(04)00507-3

[7] K. Liao and S. Li, "Interfacial Characterization of a Car- bon Nanotube-Polystrene Composite System," Applied Physics Letters, Vol. 79, No. 25, 2001, pp. 4225-4232. doi:10.1063/1.1428116

[8] K. T. Hsiao, J. Alms and S. G. Advani, "Use of Epoxy /Multiwalled Carbon Nanotubes as Adhesives to Join Graphite Fibre Reinforced Polymer Composites," Nanotechnology, Vol. 14, No. 8, 2003, pp. 791-793. doi:10.1088/0957-4484/14/7/316

[9] F. J. Gojny, M. H. G. Wichmann, U. Koupke, B. Fiedler and K. Schulte, "Carbon Nanotube-Reinforced EpoxyComposites-Enhanced Stiffness and Fracture Toughness at Low Nanotubes Content," Composite Science and Technology, Vol. 64, No. 15, 2004, pp. 2363-2371. doi:10.1016/j.compscitech.2004.04.002

[10] F. J. Gojny, M. H. G. Wichmann, B. Fiedler and K. Schulte, "Influence of Different Carbon Nanotubes on the Mechanical Properties of Epoxy Matrix Composites-A Comparative Study," Composite Science and Technology, Vol. 65, No. 15-16, 2005, pp. 2300-2313. doi:10.1016/j.compscitech.2005.04.021

[11] M. H. G. Wichmann, J. Sumfletch, B. Fiedler, F. G. Gojny and K. Schulte, "Multiwall Carbon Nanotube/Epoxy Composites Produced by a Masterbatch Process," Mechanics of Composite Materials, Vol. 42, No. 5, 2006, pp. 395-405. doi:10.1007/s11029-006-0050-3

[12] J. Zhang and D. Jiang, "Interconnected Multiwalled Carbon Nanotubes Reinforced Polymer-Matrix Composites," Composite Science and Technology, Vol. 71, No. 4, 2011, pp. 466-470. doi:10.1016/j.compscitech.2010.12.020

[13] W. C. Oliver and G. M. Pharr, "An Improved Technique for Determining Hardness and Elastic-Modulus Using Load and Displacement Sensing Indentation Experiments," Journal of Materials Research, Vol. 7, No. 9, 1992, pp. 1564-1572. doi:10.1557/JMR.1992.1564

[14] V. K. Srivastava and B. Harris, "Effect of Particles on Interlaminar Crack Growth in CrossPlied Carbon Fibre Epoxy Laminates," Journal of Materials Science, Vol. 29, No. 2, 1994, 548-553. doi:10.1007/BF01162520 